\title{
Philosophiques
}

\section{Une ou deux choses à propos de Baudrillard}

\section{Steve Light}

Volume 22, numéro 1, printemps 1995

URI : https://id.erudit.org/iderudit/027309ar

DOI : https://doi.org/10.7202/027309ar

Aller au sommaire du numéro

Éditeur(s)

Société de philosophie du Québec

ISSN

0316-2923 (imprimé)

1492-1391 (numérique)

Découvrir la revue

Citer cet article

Light, S. (1995). Une ou deux choses à propos de Baudrillard. Philosophiques, 22(1), 65-78. https://doi.org/10.7202/027309ar

\section{Résumé de l'article}

Oublier Baudrillard ? Pourquoi devrions-nous oublier un art qui, malgré sa remarquable sérénité et même sa nonchalance ostensible, s'acharne à nous dire quelque chose sur notre condition, un art qui ne craint ni d'investiguer, ni de raconter les terribles paradoxes de l'existence contemporaine et de la civilisation contemporaine, un art qui, aussi rare que cela puisse être, peut engendrer le paradoxe? d'utilisation que vous pouvez consulter en ligne.

https://apropos.erudit.org/fr/usagers/politique-dutilisation/ 


\title{
UNE OU DEUX CHOSES À PROPOS DE BAUDRILLARD
}

\author{
Steve Light
}

RESUME : Oublier Baudrillard? Pourquoi devrions-nous oublier un art qui, malgré sa remarquable sérénité et, même sa nonchalance ostensible, s'acharne à nous dire quelque chose sur notre condition, un art qui ne craint ni d'investiguer, ni de raconter les terribles paradoxes de lexistence contemporaine et de la civilisation contemporaine, un art qui, aussi rare que cela puisse être, peut engendrer le paradoxe?

\begin{abstract}
A respected commentator on contemporary French intellectual life, tells us that we should, perhaps, "forget Baudrillard $\gg$ But why should we forget such a subtle art as Baudrillard's? Why should we forget an art wich, for all its remarkable serenity and, even, ostensible nonchalance, remains impassioned to tell us something about our condition, an art fearing neither to investigate nor to narrate the terible paradoxes of contemporary existence and contemporary civilization, indeed, an art wich, rare as this is, can bear paradox?
\end{abstract}

Le fait de penser mène invariablement au malentendu. Il n'est pas surprenant, donc, que les penseurs soient mal compris. Certains plus que d'autres. Baudrillard nous parle d'un monde renversé encore plus qu'à l'envers. Mais un sociologue américain des médias, par exemple, est confondu par la catégorie de l'à l'envers. Il nous dit que Baudrillard « exalte » le sens dessusdessous. Sa remarque est faite en passant, dans un article qui n'est pas par ailleurs consacré à Baudrillard. Peu importe, puisqu'avec cette remarque, il trouve preneur. Un critique d'art va plus loin : dans un article qui devient un inventaire quintessentiel de sophismes logiques et rhétoriques, de même que des divers dogmatismes psychologiques qui sont caractéristiques de l'ignorance bêtement auto-suffisante, Baudrillard est rendu responsable du sens dessus-dessous. Mais, outre les ignorants et les illettrés philosophiques, souvent les amis ne font pas mieux. Un traducteur pense que, chez Baudrillard. c'est une question d'analyse du jeu des signes quand, en fait, l'analyse porte sur 
la logique de leur origine. Baudrillard, métaphysicien clairvoyant du tout social, est réduit au statut de sémioticien d'une seule facette de l'apparence, tout comme, jadis, un autre métaphysicien du tout social avait vu l'objet de son analyse être pris comme une forme illusoire de conscience alors qu'en fait, ce qui était en question était une relation sociale inversée mais néanmoins réelle et le système fondé sur cette inversion. On trouve une abondance d'erreurs logico-gnoséologiques que même un étudiant des classes terminales ${ }^{\mathrm{I}}$ ne pourrait se permettre de faire. L'auteur d'une étude récente sur Baudrillard, qui se veut pourtant exhaustive, ne réalise pas que l'objet de Baudrillard n'est pas le même que celui de son étude. Ainsi, Baudrillard est partout critiqué pour n'être pas arrivé à la même conclusion que l'auteur en question. Il semble que notre auteur a commis l'erreur peu élégante d'avoir cru valide une critique de Baudrillard qui le critique pour la seule et unique raison qu'il est Baudrillard! Un compte rendu récent du volume en question peut montrer l'illégitimité logique et épistémologique de ses critiques. Néanmoins, lorsque l'auteur du compte rendu énonce (avant d'énoncer ces critiques) que Baudrillard «préfère l'artifice à la nature, le pastiche à l'unité, la simulation à l'actualité... » il énonce précisément cette vision renversée de Baudrillard qu'on retrouve d'un bout à l'autre du livre que l'auteur du compte rendu cherche à dénigrer ! Effarant en effet. Mais, si on lit en ne saisissant ni les stratégies épistémologiques, ni les stratégies rhétoriques et qu'on ne saisit pas non plus ni les dispositions, ni l'ironie d'un auteur, il ne sera pas étonnant que tous les signes se retrouvent ou bien renversés ou bien éludés. Toutefois, ne devrait-il pas être évident que si quelqu'un rejette, par exemple, l'idéalisme objectif il n'est pas pour autant entraîné vers les pièges du phénoménalisme?

C'est comme ça. Un commentateur posé et respecté de l'activité intellectuelle française nous dit que nous devrions, peut-être, « oublier Baudrillard $»^{2}$. Assurément, il n'est pas entièrement sérieux. Mais la remarque ne peut qu'être assimilée - sauf par le commentateur lui-même - à une attitude intellectuelle archétypale qui, d'un côté, résidant entre autres au cœur

I. En français dans le texte [NdT].

2. Toutefois, quelqu'un dira sûrement : « Mais c'est Baudrillard qui a commencé ! » (Oublier Foucault, etc). Dans la lettre, peut-être, mais pas dans l'esprit. Baudrillard, dans son texte, prend Foucault au sérieux. Et c'est pourquoi il peut offrit le titre particulier en question. Ce titre souligne avec humour une sérieuse divergence. La réponse de Foucault - «Baudrillard ? je n'arrive même pas à me souvenir de lui » - est banalement spirituel et d'un dépit peu flatteur. Mais, mis à part la banalité et le manque de grâce, il devrait être évident que Foucault commet ici la même indiscrétion que Foucault avait jadis attribué à Sartre (Sartre ayant balayé de la main Les mots et les choses) : ne pas avoir pris la peine de lire les textes en question. 
de la tradition analytique, corrompt la réflexion philosophique - réduite qu'elle est dans cette tradition à n'être qu'un simple réflexe psychologique et, d'un autre côté, représente le cynisme prévalent qui réside au cœur de notre anti-intellectuelle civilisation moderne tardive et post-moderne ${ }^{3}$.

Mais pourquoi devrions-nous oublier un art aussi subtil que celui de Baudrillard ? Pourquoi devrions-nous oublier un art qui, malgré sa remarquable sérénité et, mème sa nonchalance ostensible, s'acharne à nous dire quelque chose sur notre condition, un art qui ne craint ni d'investiguer, ni de raconter les terribles paradoxes de l'existence contemporaine et de la civilisation contemporaine, un art qui, aussi rare que cela puisse être, peut engendrer le paradoxe? Il est dans l'ordre des choses qu'un art aussi subtil accumule les malentendus. Mais cette explication ne peut pas justifier l'incompréhension et encore moins l'ignorance dogmatique et paresseuse.

Le besoin d'assimiler Baudrillard à un milieu post-structuraliste et postmoderniste est une erreur commune au critique complaisant et à l'amical. Il est bien connu que l'anticipation peut produire des phénomènes perceptifs. Si on cherche Baudrillard là où il n'est pas, naturellement on l'y trouve. Toutefois, il arrive un moment où, pour comprendre une forme de pensée, on doit arriver au lieu de son intuition. Mais on doit aussi pouvoir déterminer la raison pour

La polèmique est un art des plus difficiles. Puisqu'une polémique ne peut être digne de ce nom que si elle considère quelque chose sérieusement, c'est-à-dire si elle réussit à attribuer à son objet tout le crédit (aussi petit soit-il) auquel cet objet a droit. Même la stupidité affirme « quelque chose ». Mais, si ce «quelque chose » n'est pas constitué à l'intérieur de la polémique, il n'y aura rien contre quoi polémiquer.

3. Les efforts récents en Allemagne et en France pour rehabiliter une tradition cynique authentique et originale peuvent-ils échapper au simple formalisme pour éviter de renforcer certaines tendances d'une civilisation cynique dont le pouvoir de récupération a largement été sous-estimé par nos réhabilitateurs? En fait, la réhabilitation et l'adoption de toute tradition authentique et en particulier d'un courant de pensée antique sera, s'il est authentique, préférable aux modes de pensée prevalents. Après tout, les traditions originales incorporent, par définition, une sagesse authentique. Diogène n'a-t-il pas des « côtés » que Zénon ou Épicure ou, pourquoi pas, que Chrisippe ou Sextus n'ont pas? Mais l'inverse est aussi vrai. La réhabilitation requiert que les « côtés » soient remplis. Et c'est pourquoi, sans même devoir recourir à Hegel, il y a une certaine indifférence dans le choix d'un réhabilitateur. Mais la question est justement là. Nous avons tendance (inévitablement mais, sans doute avec raison) à reporter dans le temps les solutions aux problèmes contemporains. Pourquoi parlons-nous du formalisme? Parce que la plupart des réhabilitations cherchent à emprunter un raccourci vers notre sagesse, elles la recherchent toute-faite, ce qui est justement la forme dans laquelle elle ne peut pas, par définition, être recherchée. 
laquelle c'est à cet endroit précis qu'arrive l'intuition. Aussi, on doit s'efforcer de voir ce qu'une forme particulière de pensée voit. Assurément, Baudrillard partage la culture intellectuelle du post-structuralisme ${ }^{4}$, mais il ne pose pas les problèmes spécifiques à cette culture comme son point de départ comme le font Derrida et Lyotard (et même, à sa façon, Deleuze). Si Baudrillard se distingue, c'est parce qu'il est (et doit être vu comme étant) l'unique disciple authentique de la critique situationniste de la culture et de la civilisation du capitalisme tardif ${ }^{5}$. C'est cette position, toutefois, qui rend son assimilation aux conceptualisations post-structuralistes et post-modernes philosophiquement illégitime. Lyotard est un théoricien post-moderne (il n'est donc pas un théoricien $d u$ post-moderne) et c'est pour la simple raison qu'il croit que la réalisation est simultanément toujours possible (il croit en la perpétualité des avant-gardes et, dans cette position - sûrement ironique - reste aussi vulnérable - ne possédant aucune critique adéquate de la récupération - que l'est Habermas devant une réalité qui partout déjoue l'indulgence théorique de l'un (Lyotardl, et la magnanimité théorique de l'autre (Habermasl) et qu'elle l'est plus qu'elle ne l'était. La soi-disant « chute des grand narratifs » (qui peut être vue comme l'un des aspects de la transition d'une ère de crise culturelle à l'état actuel d'impasse absolue, quand elle est traduite dans un langage plus cohérent et, donc, située dans une analyse plus cohérente) constitue pour Lyotard une libération paisible ${ }^{6}$.

Le cercle des lecteurs visés par Baudrillard est, toutefois, beaucoup plus circonscrit. Comment doit-on penser une société, une culture, une civilisation qui « a manqué le moment de réalisation »? La formule, qui est d'Adorno, fixe

4. De la même façon que tous les penseurs marquants d'une période donnée partagent une certaine culture, étant, en vertu de leur importance, de leur temps.

5. Notre emploi du mot « disciple » doit être bien comprise. Il y a plusieurs sortes de disciples. Dans le contexte présent, nous parlons d'une relation objective (logique), et non subjective. Baudrillard (de deux ans l'ainé de Debord et de cinq ans le cadet de Vaneigem, par exemple) n'a jamais fait partie du groupe situationniste et ne partageait pas non plus la culturé et la position immédiate du groupe. Il n'a d'ailleurs pas été formé par les thèses situationnistes. Si Baudrillard a toutefois partagé le site existentiel et théorétique du radicalisme situationniste, c'est aussi Baudrillard qui a réagi de la manière la plus cohérente et la plus radicale à l'impasse intellectuelle et théorique atteinte par tous ceux qui avaient mené la critique radicale de la modernité tardive à sa conclusion la plus cohèrente et la plus radicale.

6. De toute évidence, on ne peut pas dire qu'il y a une connexion nécessaire entre nos theoriciens post-modernes et les diverses complicites (les justifications intellectuellement paresseuses et les prises de positions auto-complaisantes auxquelles certaines âmes, inexplicablement, semblaient avoir adhéré) qui ètaient évidentes dans l'appui intellectuel à la guerre du Golfe mais, assurement, les deux positions ne sont pas du tout inconsistentes l'une avec l'autre. 
(implicitement) le moment, rétrospectivement et symboliquement à 1918. C'est ici que la modernité a eu sa dernière chance de se tracer une nouvelle voie, d'invalider la thèse Wébérienne de l'unilatéralisme. Mais le mouvement des travailleurs fut défait, et la formule pour cette défaite est bien connue : 1917Igr8-I922-Ig27-1933-1937. La pensée di'Adorno est formée par la marche du nonretour de la modernité. Ayant déjà montré, dans La dialectique de lillumination, son intuition de la structure et de la forme de la modernité tardive qui s'est développée aux États-Unis au cours de la période qui a suivie la Seconde Guerre (laquelle modernité tardive ne fut exportée en Europe qu'après ig68), la pensée d'Adorno est fidèle à son auto-caractérisation comme « message de détresse des naufragés ». La génération subséquente, du fait qu'elle possède une formation différente, doit toutefois arriver à cette intuition par elle-même. Conséquemment, cette intuition est le résultat d'une attitude et d'une position qui sont caractéristiques des avant-gardes (politiques et théoriques) activistes, c'est-à-dire Socialisme ou Barbarie, L'Internationale Situationniste, plutôt que d'être le résultat de l'attitude et de la position relativement plus contemplatives de l'avant-garde philosophique d'où est issu Adorno. En fait, Castoriadis, en se basant sur une méditation encyclopédique de notre modernité, libère la philosophie sociale des obstacles issus de la philosophie classique allemande hèritée par Marx et, donc, développe la philosophie la plus attirante du projet humain et moderne ${ }^{7}$. D'un autre côté, la critique situationniste est le dernier non! de l'avant-garde au parti unilatéral et irrévocable que la modernité a adopté. Ainsi, 1968 peut constituer la dernière apparition de 1918 (la dernière lueur d'une étoile disparue), comme le non!

7. Nous savons bien que Castoriadis contesterait notre conception selon laquelle il est le seul vrai disciple de Marx, un disciple en vertu du fait que le developpement par Castoriadis d'une philosophie sociale cohérente a, en fait, aussi rendu la philosophie sociale de Marx cohérente. Ce que La Critique de la raison dialectique, L'Ontologie de lêtre social et La Reconstruction du matérialisme historique n'accomplissent pas, L'Institution imaginaire de la société l'accomplit. Sans doute, Castoriadis a eu à combattre des tentatives pour l'associer à Marx. Toutefois, Castoriadis a été l'héritier privilégié de l'échec de Marx à rendre compte de manière cohérente du projet humain et de la lutte humaine. A cause de cethéritage, à cause du fait qu'il a repris le même problème et, donc, l'a repensé, Castoriadis a reconstitué la manière de penser le projet et la lutte en question. Mais dans cette perspective, Castoriadis n'est pas moins dans la foulée de Marx que Spinoza, par exemple, n'est dans la foulée de Descartes, étant entendu, toutefois - et comme Henri Gouhier l'a fait remarquer avec sagacité - que le spinozisme n'est pas le résultat logique du cartésianisme (cette notion n'étant, comme le remarque Gouhier, qu'un concept polémique introduit par Leibniz). Si un certain Baruch n'était jamais né, il n'y aurait jamais eu de spinozisme. D'un autre côté - et c'est notre point principal ici - si un certain René n'était jamais né, le spinozisme n'aurait pas pris la même forme. 
situationniste peut constituer la dernière apparition de 1936-37. On avait irrévocablement manqué le moment de réalisation.

Si, donc, Adorno était le penseur du côté négatif de cette formule, il restait à Baudrillard à penser le côté positif (au sens toutefois, de positivité pure). La problématique révolutionnaire de Adorno, de Castoriadis et des situationnistes, qui est aussi, biographiquement et théoriquement (en vertu de ses premiers travaux) celle de Baudrillard, devient maintenant, dans sa variation nécessairement transformée de problématique de l'impasse civilisationnelle, celle de Baudrillard seul. Adorno est mort trop tôt. Les situationnistes concluent avec leur non ! et quoique Castoriadis énonce effectivement la problématique, il ne le fait qu'en passant, son attention étant portée strictement vers la philosophie sociale.

Comment éviter la voie dans laquelle la modernité s'est irrévocablement engagée. Jusqu'à la parution de son ouvrage de 1976, LÉchange symbolique et la mort, Baudrillard façonne, au sein de la critique radicale de la modernité tardive toutes les précisions qu'entraîne nécessairement la transition amorcée vers la post-modernité, tout en préservant, dans sa réflexion, le dynamisme du non! situationniste. Mais, avec l'avènement de la post-modernité proprement dite, on comprend que la formule Adornienne doive être revue. Il faut comprendre que Baudrillard ne fait pas lui-même référence à la formule d'Adorno. Non seulement le moment de réalisation a-t-il été raté, mais, en vertu du fait qu'il a été raté, et précisément en vertu de ceci seul, il a - aussi - été réalisé ! Baudrillard devient donc le cartographe de cette réalisation paradoxale. Comment quelque chose peut-elle être réalisée en vertu du fait qu'elle n'est pas réalisée ou ne pas être réalisée en vertu du fait qu'elle est réalisée ? C'est à cette question que Baudrillard a tenté depuis les dix dernières années de répondre de différentes façons pendant que le cours de la (post) modernité s'est accélérè. Baudrillard avait dejà, bien sûr, confronté cette question dans le cadre de ses réflexions antérieures (parce que la post-modernité « réalise » la modernité tardive $^{8}$. Toutefois, la spirale de notre modernité a maintenant dépassé les complexités de la récupération en soi. Une ceuvre telle que La Transparence $d u$ mal peut donc commencer par : « Aujourd'hui tout a été libéré, tous les jetons joués et nous nous trouvons collectivement face à la question cruciale : que

8. Il suffit de penser aux analyses de Baudrillard sur le corps dans La Société de consommation par exemple. Il est d'ailleurs triste que les analyses incisives et subtiles du corps exposées par le sociologue Japonais Osamu Mihashi dans son ouvrage de 1983. Tobenai karada (Le Corps qui ne peut pas volent demeurent, pour des raisons linguistiques évidentes, inaccessibles aux lecteurs occidentaux. Il est plus triste encore que ses analyses sur le sexe, la culture et la société dans son «Konchikusho» $k o$ : edo-no shinseishi (Sur «Konchikusho » : une histoire de la mentalité de la période Edo, Tokyo, Nihon edita sukuru shuppan-bu, 1992) le soient aussi. 
faire après l'orgie? $\gg^{9}$. On pourrait dire que c'est précisément cet « après » qui représente la pleine émergence de la post-modernité et qui signifie que nous avons quitté pour de bon la période de transition entre la modernité tardive et la post-modernite.

Auparavant, Baudrillard avait utilisé une périodicisation dans laquelle une description de trois phases successives de la valeur illuminaient la succession du traditionnel au moderne et du moderne au moderne tardif : « un état naturel d'état-valeur, un état de commodité de valeur-échange, un état structurel de valeur-signe $\gg^{\text {To }}$. Mais, avec l'arrivée de la post-modernité en tant que telle, il est nécessaire de parler d'une quatrième phase, d'un niveau « fractal » de valeur. Peut-on trouver une meilleure métaphore pour illuminer une contemporanéité caractérisée d'abord et avant tout par le débordement et le mélange des genres, par un confusionnisme généralisé des catégories? Le sexe n'est plus dans le sexe, le politique n'est plus dans le politique. Ils traversent tout ( $\ll$ un degré xerox de la culture $»^{\text {II }}$ raille Baudrillard). Nous assistons à une destruction totale du catégoriel au profit d'une sorte d'hypersyncrétisme du sans-relations. Et c'est pourquoi Baudrillard peut faire un usage métaphorique si clairvoyant de la métastase. Le fractal représente le sans-référence, l'auto-reproduction à l'infini, la liquidation par l'excès : un processus viral (mais, donc, virulent!) d'indistinction. Qu'est-ce que l'« extase de la communication »? Par la destruction de toutes préconditions à une quelconque part intersubjective, cette « part» est « réalisée » par ce qui pourrait s'appeler un circuitage de masse de l'atomisé. Un pas-du-tout devient un trop- $d u$-trop en vertu de l'accélération d'une connexion qui n'en est pas une. L'imaginaire du miroir, le dédoublement de la scène, l'aliénation de l'altérité cèdent le pas à l'écran et à l'interface avec leurs redoublements, leurs contiguïtés et leurs réseaux. Ce qui était traversable par le corps (étant donnée la distance du langage, de la scène, etc.) cède la place à l'écran intraversable. Qu'estce que la «communication »? L'abstraction multipliée. Ce n'est pas l'aliénation, mais plutôt un circuit intégré qui est le principe privilégié ici. Et qu'est-ce que le medium ? La multiplication des formes virales. Leur multiplication et leur accélération. Et ce n'est pas tant parce que la maturation et le développement donnent lieu à l'instantanéité, ni que le en-soi a lui-même été annihilé. Parce que ce n'est pas la matérialisation d'une absence, mais bien celle de la pure positivité de l'abstraction. Une multiplication de l'insensé.

Pour décrire cette multiplication et cette accélération et ces « réalisations immédiates », pour décrire ce post-catégoriel du «post-orgie », on doit faire

9. Jean Baudrillard, La Transparence du mal Paris, Grasset, 1990, P. I.

Io. Ibid., p. 13.

II. Ibid., p. I6. 
appel à une tératologie sociale qui n'aurait guère pu être imaginée jusqu'à maintenant. Baudrillard traque les « réalisations » en question à travers tous les domaines de la modernité et, pour leurs instanciations post-modernes. invente le «trans-politique », le «trans-économique » le «trans-esthétique » etc. Nous assistons à un mouvement de la transcendance à l'« orbital », d'un état de crise à un état de fatalité et d'extrémité. Qu'est-ce que le « transéconomique »? «La transcendance de l'économie par ses propres simulacres $\gg^{{ }^{2}}$. La destinée du capital devient le modèle privilégié. Le capital a échappé à sa propre fin (tout comme l'économie a échappé à la réversibilité de la crisel. Qu'est-ce que la « réalisation immédiate »? Que veut dire le «trans- » de «trans-politique », «trans-économique » etc. ? Il s'agit de l'habileté de quelque chose à fonctionner « au-delà de sa propre finalite $»^{13}$. Puisque toutes les fondations référentielles ont été détruites, il n'y a rien pour empêcher cette auto-reproduction à l'infini qui est caractéristique du jeu pur. Il s'agit là de la «perfection et la dénigration » simultanées de la modernité. Une liquidation par l'excès. Mais il s'agit de l'excès de la « saturation » nous dit Baudrillard, et non pas de l'« excès critique » de Bataille. Le moment de réalisation a été raté? Toutefois, par une sorte d'écrasement et d'accélération, par une sorte de ventriloquisme matériel, la « réalisation » devient la vitalité pure de l'inertiel lui-même. Mais ce n'est pas une question d'une stabilité en elle-même. Cette auto-reproduction sans référence n'est rien de moins qu'une « révolution de $l^{\prime}$ incertitude $»^{14}$ parce que l'« incertitude résulte d'un excès de positivité $»^{15}$. Raison de plus qui fait que le «système entier Idevient visiblementl terroriste. $\gg^{{ }^{6}}$. Nous n'avons plus la domination par le code, mais plutôt la domination par l'accélération d'un code comprimé, une sorte d'hypertrophisme d'une « exacerbation du code $»^{17}$. Saturation ? Pure positivité, ce «manque incestueux d'altérité $~^{18}$ est lui-même « la transparence du mal ». Pure positivité : toutes choses perdent leur substance, leur distance, leur résistance. L'indifférence totale de l'accélération et l'accélération de l'indifférence totale : autrefois le «principe mauvais » signifiait le «contre- » immédiat, maintenant ce n'est que l'« altérité secrète de la viralité ${ }^{19}$, la perméabilité absolue. Passant au travers de toutes choses, elle est, si on veut,

\footnotetext{
I2. Ibid., p. $3^{8}$.

13. Ibid.

14. Ibid., p. 49.

15. Ibid., p. 59

16. Ibid, p. 49 .

I7. Ibid.

I8. Ibid., p. 72

I9. Ibid.
} 
disparue en elles, signe illisible d'une abjection absolue et de la fatalité du système.

Nous pouvons à peine imaginer toutes les subtilités d'une tératologie sociale. C'est Baudrillard, sans doute, qui nous les a données. Il a lui-même fourni la devise de La Transparence du mal : « Puisque le monde conduit à un état délirant des choses, nous devons nous conduire vers un point de vue délirant. » Dans cette devise réside tous les secrets de l'art de Baudrillard, de même que toutes les raisons pour lesquelles la méprise prolifère tant autour de ses analyses. La destruction du catégoriel. La nullification de toute relation. Le bris de la cause et de la fixité. Les fondements de toutes les stratégies épistémologiques antérieures ont été minées, ce qui n'est que l'envers du fait qu'il n'y a plus de protection de cet « espace symbolique et mental » par lequel la faculté de juger pouvait exister et fonctionner. Savoir a toujours voulu dire donner un poids convenable à toute chose. Mais dans l'état actuel d'extrémité catastrophique, comment peut-on donner un poids à des choses qui ne sont même plus des choses ? Comment donner un poids à cet écrasement sans pesanteur de l'accélération et de l'indifférence? Adorno dit que dans la psychanalyse, seules les exagérations sont vraies. Non seulement l'essence peut-elle se cacher mais l'apparence aussi. Aujourd'hui, le système ne cache rien cependant. Il est visiblement et globalement une exagération. Toutefois, par le fait même il est devenu la banalité en-soi. En quoi pourrait donc consister une nouvelle métaphysique du réel ? Baudrillard a parlé, dans L'Échange symbolique et la mort, de la nécessité de créer une "science fiction du système se détruisant lui-même », d'une «science des solutions imaginaires ». La transition du moderne tardif au post-moderne impose sans aucun doute une révision de cette stratégie (en font foi les «stratégies fatales » de Baudrillard). Comment donc peut-on révéler l'exagération extrême du système? C'est comme si toute description devrait devenir une sorte de « science de la description imaginaire » lequel « imaginaire » est, paradoxalement, le seul à pouvoir fournir le visagelittéral du réel. Ne pourrionsnous pas dire que la virtualité du système est plus actuelle que sa propre actualité ? faire en sorte que le système prenne ses « descriptions imaginaires » littéralement, parce qu'il est littéralement si extrême, si terrible? En un sens, ce n'est pas Baudrillard qui exagère autant que le système qui, par la vitalité de sa reproduction incessante, exagère sa propre banalité, ce qui oblige à redonner au système son propre etat exagéré.

Baudrillard a bien sûr parlé de ses «fictions», et il a lui-même utilisé le terme « science fiction ». Ceci est, en un sens, regrettable puisque cela a ouvert le chemin à l'assimilation et à la (tentative de) récupération de Baudrillard par les post-structuralistes. Mais on ne doit pas oublier que, chez Baudrillard, le matériau de la « fiction » et de la « fictionalisation » est sociologique et, par 
ailleurs, que la «fiction » en question est le produit d'une stratégie philosophico-épistémologique rigoureuse qui cherche à confronter directement les dilemmes et les énigmes que notre post-modernité pose au projet historique d'une théorie (et d'une position) sociale critique (ou non complice, du moins aujourd'hui).

Le dilemme terrible, toutefois, est qu'une restauration théoricoconceptuelle de ses états exagérés au système requiert un processus d'établissement du sens qui peut devenir inextricablement emmêlé avec (et, donc, récupérable par) la logique d'un système qui est devenu surplus de sens absolu (et absolument accéléré) qui est, bien sûr, aussitôt une absence absolue. Donc, l'établissement théorique du sens en question, un établissement de sens sans lequel il ne peut y avoir aucune entreprise théorique, encore moins une quelconque entreprise théorique radicale, se trouvera nécessairement poussé (et, donc, menacé !), par cette logique de l'accélération par laquelle le système connaît sa propre auto-reproduction à l'infini. Cest comme si toute analyse qui chercherait une relation radicale au réel, qui chercherait, si on veut, à rattraper le réel (ou, du moins, à ne pas se laisser dépasser par lui, lequel réel n'étant pas autant réel - n'étant plus maintenant que l'instanciation d'une hyperréalité - que terriblement réel) nous forçait à avoir recours à une sorte d'« hyper-établissement de sens ». Il y a donc un danger que l'analyse subisse elle-même une sorte de saturation ${ }^{20}$. Il y a un danger qu'il advienne une symétricalisation du réel et de sa métaphysique. Toutefois, ce danger est le danger nécessaire, la précondition nécessaire à toute analyse radicale authentique. Il y a de toute évidence chez Baudrillard une certaine «fascination » pour l'extrémité du système, pour les «phénomènes extrêmes et délirants » de l'accélération du système. Mais l'erreur classique de trop de critiques et de lecteurs de Baudrillard (qu'ils soient critiques ou sympathiques) est d'avoir pris cette « fascination » (qui n'était, dans aucun cas, reconnue en des termes qui lui convenaient) pour une marque de ratification. Mais ce qu'on oublie ici est, d'une part, que la fascination en question est une condition de la promulgation de la stratégie épistémologique radicale à laquelle elle est rattachée (parce qu'il n'y a qu'elle pour nous empêcher de tourner le dos à ce réel terrible ou d'avoir simplement recours à la position tragique du «à quoi bon? ») mais aussi, et de manière plus fondamentale, que cette fascination est accessoire à une stratégie

20. Une « saturation » qu'en vertu de leur « productivisme », les analyses de Foucault non seulement n'arrivent pas à éviter, mais entretiennent partout et nécessairement (et entretiennent d'autant plus, en vue de la virtuosité de Foucault), ainsi que Baudrillard l'avait très tôt fait remarquer et qu'il avait subséquemment résumé d'une manière si clairvoyante dans Oublier Foucault. 
épistémologique qui n'est elle-même, comme stratégie, que le résultat d'une répulsion fondamentale.

Toutefois, la stratégie de Baudrillard a nécessité la suppression de l'expression subjective de cette répulsion. Il y a, chez Baudrillard, une askesie théorique radicale. Le « rejet » critique, le « rejet » que postule nécessairement le criticisme, ne peut plus aujourd'hui se loger aussi facilement dans une analyse radicale parce que le système est devenu plus radical que le criticisme lui-même. C'est à ce dilemme que répond l'askesie de Baudrillard. Toute analyse qui n'exprime pas suffisamment la radicalité du système sera sujette à toute la gamme des faux espoirs libéraux/rationnels et/ou à toutes les nostalgies paresseuses qu'on peut trouver (en opposition, devrions-nous noter - et un peu contre Baudrillard lui-même - à ce qui doit être considéré la nostalgie radicale de Adorno). Et ce sont précisément ces attitudes que l'ascétisme théorique que Baudrillard s'impose est censé combattre. Bien sûr, demeure le danger (encore une fois il s'agit du danger de « symétricalisation » duquel nous parlions plus tôt) que l'askesie en question - cet effacement tout court, dans l'analyse théorique, de toute marque de la position subjective qui a donné lieu, et qui seule peut donner lieu, à cette analyse - laisse un « silence théorique » au centre de la métaphysique en question, et que ce silence ne suffise pas à contrer cette virtualité et cette positivité qu'il ne peut, en fait, tolérer ou endurer et qu'il devienne le conduit par lequel elles entrent dans l'analyse. Mais ce danger n'est-il pas bien moindre que le danger auquel les critiques de Baudrillard s'exposent? Les critiques libéraux et particulièrement les critiques de gauche ne se retrouvent-ils pas avec ce qu'on pourrait appeler une « utopie de la répulsion », justement parce que leur opposition subjectivo-rationnelle les a empêchés de tenir suffisamment compte de la répulsivité absolue du réel (répulsivité que la stratégie de Baudrillard cherche à amplifier en faisant en sorte que c'est le système lui-même qui peut être amené à affirmer sa propre extrémité littérale) ? Nest-il pas immédiatement évident (péniblement évident) que les critiques de Baudrillard finissent toujours par réduire la philosophie et les réflexions théoriques au positivisme pré-philosophique du « tout ne va pas si mal » conversationnel ? Et ne prêtent-ils pas leur voix à ce genre précis de « ratification » qui est toujours inhérente aux faux-optimismes, ce genre précis de « ratification » que l'ascétisme théorique de Baudrillard (disons, plus fondamentalement, sa « répulsion ») est censé combattre?

Une fois de plus nous devons dire que c'est la stratégie philosophicoépistémologique de Baudrillard qui est mal comprise, et c'est dire que beaucoup des critiques de Baudrillard sont simplement et bêtement illettrés philosophiquement. C'est pourquoi certains aspects des «fictions» de Baudrillard sont, à tort, pris litteralement alors que d'autres ne sont pas pris littéralement justement quand ils devraient l'être. C'est également pourquoi les critiques (le commentateur génial auquel nous avons fait allusion plus tôt 
ne fait pas exception) peuvent si injustement l'accuser de s'accomoder politiquement du statu quo. Ayant, d'une part réduit la réflexion de Baudrillard à une méditation post-structuraliste sur la signification et le sens et ayant, d'autre part, réduit sa théorie sociale à une simple sémiologie d'une des facettes de l'apparence, et, donc, n'ayant pas réussi à cerner la manière dont les « critiques » épistémologiques et sociales forment une unité stratégique chez Baudrillard (n'ayant pas, en d'autres mots, réussi à atteindre l'intuition originale et l'axe philosophico-conceptuel de la pensée de Baudrillard, et, en plus, n'ayant pas, semble-t-il, réussi à comprendre que même l'empirisme luimême a progressé, il y a une génération déjà, vers une compréhension de l'incommensurabilité théorique), ses critiques (et le critique/commentateur mentionné plus tôt) pensent qu'ils peuvent invalider l'analyse apparemment « sombre » et « accomodationniste » de Baudrillard en montrant du doigt des « réalités réelles », quand, en fait, ces « réalités réelles » n'existent pas en dehors de leur réflexion analytique particulière. Et, qui plus est, ils n'ont pas compris que, dans le cas présent, les « réalités » précises qu'ils montrent du doigt ne peuvent exister que dans l'analytique (modelée de façon cohérente) d'une économie politique du réel et non pas dans l'analytique d'une métaphysique du réel. Une fois de plus, nous comprenons à la fois la nature et l'origine de ces critiques illégitimes, nous comprenons que Baudrillard est critiqué pour la seule raison qu'il est Baudrillard, comme s'il était légitime de critiquer A pour la seule raison qu'il n'est pas non-A.

Il y a quelque dix ans, Pierre Vidal-Naquet, évoquant les divers types de critique sociale, a fait allusion aux critiques des troisième-ordre comme celles des situationnistes et de Baudrillard. Mais hormis Vidal-Naquet, on ne trouve chez aucun commentateur de Baudrillard la compréhension de cette différence plutôt évidente à laquelle nous faisons allusion, à savoir, la différence entre les divers niveaux d'abstraction et entre les niveaux de réflexion analytique. Il ne devrait donc pas être surprenant qu'on comprenne si mal, après tout, qu'une division du travail intellectuo-conceptuel est nécessaire (mais pas nécessairement contradictoire), mais aussi qu'il existe plus d'un « monde ». Le fait que le capital existe aujourd'hui au-delà de sa propre finalité ne veut pas du tout dire que le capital n'existe plus. Et ce fait ne représente pas tellement une contradiction que le paradoxe nécessaire de notre modernité tardive et de notre post-modernité. Mais ce n'est que par l'abstraction caractéristique d'une métaphysique du réel qu'on peut espérer obtenir la « vérité » à propos du paradoxe en question. En effet, ce paradoxe ne peut même pas apparaître en dehors de l'abstraction en question. En contre partie, il faut insister sur le fait que les analytiques d'une économie politique du réel et d'une métaphysique du réel ne s'opposent pas nécessairement. Il importe peu que leurs points d'arrivée théoriques exigent ou puissent exiger des attitudes pratiques apparemment différentes (parce que le monde est - vieille vérité - à la fois 
unique et multiple). La « vérité » d'une métaphysique du réel est-elle d'un ordre supérieur à la « vérité » d'une économie politique du réel ? Logiquement, elle l'est sans doute. Mais les vérités des deux niveaux sont nécessaires. Toutefois, la «vérité » qui nous est donnée par une métaphysique du réel change effectivement notre compréhension du statut de la " vérité » d'une économie politique du réel, et donne à cette dernière une « vérité » qu'elle ne pourrait pas obtenir par elle-même lc'est pourquoi le processus inverse ne peut pas avoir lieu). Toutefois, si une métaphysique du réel nous donne une vision plus englobante, ce serait une erreur de penser que cette métaphysique pourrait, ce faisant, nier la nécessité de la « vérité » d'une économie politique du réel. Les commentateurs de Baudrillard et ses critiques ont commis une double erreur. Premièrement, et comme nous l'avons déjà dit, ils n'ont pas reconnu les niveaux différentiels des « vérités » et des analytiques en question (c'est-à-dire leur incommensurabilité logique) et, deuxièmement, ils ont faussement imputé à Baudrillard cette erreur selon laquelle la nécessité des deux vérités serait niée.

En somme, le paradoxe de notre modernité tardive et de notre postmodernité doit être vécu, c'est-à-dire qu'il ne doit pas être réduit (et donc dissimulé) à avoir le statut d'une contradiction qui peut alors être trop vite résolue. avec comme résultat le fait qu'on peut éviter les vrais dilemmes de notre existence contemporaine théorético-sociale et morale. La métaphysique baudrillardienne du réel ne dit pas tant que le monde (que le système du capital) a disparu mais surtout que l'« accélération » que connaissent maintenant le monde et le système rend toutes les améliorations sociales nécessaires sujettes à des énigmes et des difficultés sans prècédent (et il faut dire que cette notion de «difficulté » ne serait qu'une notion banale sans l'élaboration que Baudrillard fait de ces énigmes et de ces difficultés, parce que, une fois de plus, à l'origine (objective) de la réflexion de Baudrillard, il y a une motivation critico-politique ). Paradoxe? Non, puisqu'il n'y a pas (et, sans doute, jamais eu) de contradiction nécessaire entre, d'une part, l'existence d'une activité sociale ou d'un activiste social (ou travailleur social) ou du processus quotidien d'amélioration sociale et, d'autre part, l'existence d'une activité philosophique ou d'un philosophe ou du processus d'abstraction socio-théorique. Par contre, il va de soi qu'il y a un choix à faire, même si on peut aussi trouver des manières de choisir les deux let non seulement parce que la temporalité peut, après tout, résoudre ce qui semble être une contradiction logiquement insoluble). Mais la manière, c'est partout ce qui compte le plus. Parce qu'on peut chosir de manière contradictoire et on peut choisir d'une manière noncontradictoire (ce qui revient à dire qu' on peut chosir de manière cohérente ou non-cohérente). Mais seul un examen de la «manière » (et un qui est pratiqué d'une certaine manière !) peut nous dire de quelle manière un choix a été fait et de quelle manière de choix il s'agit. Ce serait bien si nos critiques 
contemporains pouvaient reprendre la manière de Baudrillard d'une façon qui ne soit pas aussi sans-manières (parce qu'il va sans dire qu'un manque de manières peut faire disparaître très rapidement toutes les préconditions de l'intelligence et de la compréhension intelligente). Hélas! Nous savons très bien que c'est le manque de manières qui est la manière de plusieurs critiques de notre vie intellectuelle contemporaine. Et c'est précisément l'étendue de ce manque de manières du monde (ou du système) que Baudrillard expose avec tant de pénétration, d'audacité et de verve, c'est-à-dire d'une manière si géniale et subtile.

(Traduit de l'anglais PaR Gilles SaINT-Louis)

Etudes interdisciplinaires

Université de Californie Berkeley 